\title{
La formation à la recherche au cours des études médicales: quelques considérations, quelques questions et quelques pistes
}

\author{
Research training during medical studies: some insights, some questions \\ and some avenues
}

Une contribution originale proposée dans ce numéro par Zhang et al. [1], issue d'un travail collaboratif sinobelge, aborde la problématique de la formation à la recherche des étudiants en médecine. Les auteurs rapportent les résultats d'un travail visant à documenter l'impact d'une formation dédiée au développement de compétences en recherche, organisé au cours d'un programme de premier cycle des études médicales. Ils exposent les bases conceptuelles et les modalités opérationnelles d'une intervention pédagogique conçue selon le format de l'apprentissage en équipe (team-based learning), organisé sous la forme de trois sessions de travaux en groupes, de trois heures chacune, succédant sur une période d'une semaine à un travail personnel. Nonobstant certaines limites, ils montrent que, dans le cadre d'un tel dispositif, les activités d'apprentissage partagées entre différents groupes d'étudiants, dont les ressources respectives sont mises en commun, ont eu un impact positif sur le développement des capacités nécessaires pour rédiger un projet de recherche, individuellement ou en groupe. De tels résultats fournissent assurément des pistes encourageantes, de nature à aider les responsables des programmes à répondre, dans les différentes institutions, à une injonction désormais ubiquitairement formulée.

\section{De nombreuses recommandations internationales convergentes}

La formation à la recherche au cours de la formation médicale initiale fait en effet l'objet de nombreuses recommandations internationales, globalement convergentes, qui en argumentent l'importance et la nécessité pour tous les étudiants, quelles que soient leurs futures disciplines d'exercice.

À un niveau très général, c'est par exemple le cas, en Europe, des préconisations formulées en lien avec le processus de Bologne, mis en œuvre pour construire l'espace européen de l'enseignement supérieur. Prescrivant une architecture unique en trois cycles d'études des parcours d'enseignement supérieur (bachelier - ou licence-, master, doctorat), ses dispositions indiquent que chacun des trois cycles, dès les deux premiers (qui équivalent à la formation médicale dite pré-graduée dans certains pays), doit inclure une formation à la recherche [2].

Dans le champ spécifique de la formation médicale, la World Federation for Medical Education recommande que les capacités et dispositions à conduire un travail de recherche soient explicitement désignées comme des résultats attendus à l'issue de la formation médicale et que, tout au long du curriculum, soient enseignés les principes de la méthode scientifique, incluant la pensée analytique et critique, et les méthodes de la recherche médicale [3].

$\mathrm{Au}$ Royaume-Uni, la dernière mise à jour en 2018 [4] du cadre de référence Outcomes for graduates (Tomorrow's Doctors) publié par General Medical Council (GMC) [5] indique explicitement qu'à l'issue du programme prégradué, l'étudiant devrait être en mesure de « formuler des questions de recherche simples en sciences biomédicales, psychosociales et des populations, et de concevoir des études ou des expérimentations pour instruire ces questions». Le référentiel de compétences pour les programmes postgradués (Generic professional capabilities framework) [6] précise davantage les capacités requises, en indiquant que le diplômé devrait « démontrer sa connaissance appropriée des méthodes de recherche, incluant les approches qualitatives et quantitatives de l'investigation scientifique, ainsi que [...] des principes de la recherche et de sa transposition dans la pratique, incluant le recrutement dans des essais et programmes de recherche, ainsi que les implications éthiques liées à la gouvernance de la recherche».

$\mathrm{Au}$ Canada, les normes du Comité d'agrément des facultés de médecine du Canada [7] stipulent que les programmes prégradués «comprennent un enseignement relatif à la méthode scientifique et aux principes scientifiques et éthiques fondamentaux de la recherche clinique et translationnelle, y compris [quant à la] façon 
[selon laquelle] ces activités de recherche sont menées, évaluées, expliquées aux patients et appliquées au traitement des patients ». Pour les programmes gradués, les prescriptions sont formulées dans le référentiel de compétences CanMEDS [8] en lien avec la caractérisation du rôle d'érudit. On y indique notamment que le diplômé doit «faire preuve d'une compréhension des principes scientifiques de la recherche et de l'enquête scientifique, [...] reconnaître les principes éthiques de la recherche, [...] contribuer aux travaux d'un programme de recherche, poser des questions de recherche pertinentes et choisir les méthodes ».

En Suisse, le nouveau référentiel de compétences PROFILES [9], qui remplace depuis la rentrée 2018 l'ancien Catalogue suisse des objectifs, reprend explicitement les rôles énoncés dans CanMEDS. Il caractérise donc également le rôle d'érudit, en précisant notamment que le médecin diplômé devra être capable de «comprendre les principes théoriques généraux de la connaissance médicale et scientifique [...], d'identifier et de développer une hypothèse ou une question de recherche, d'élaborer une procédure permettant de répondre au problème, d'analyser et de synthétiser les résultats, et de les publier dans le cadre d'un rapport de recherche ou d'un article scientifique».

En France, les objectifs visés à l'issue du second cycle des études médicales décrivent de façon similaire sept compétences dites génériques, parmi lesquelles la compétence scientifique, au regard de laquelle on prévoit que l'étudiant «participe à la formulation d'une problématique de recherche face à une situation non résolue et envisage les pistes susceptibles de résoudre cette problématique » [10]. Dans le cadre des diplômes d'études spécialisées de troisième cycle, dont les maquettes ont été profondément révisées en 2017 [11], les capacités exigées sont formulées de façon différenciée selon le moment du cursus. Ainsi, à la fin de la phase 2 dite d'approfondissement, l'interne doit notamment être capable «[...] d'évaluer les méthodologies et critiquer les conclusions d'études cliniques, de participer à la rédaction d'un protocole de recherche clinique, de présenter les résultats d'un travail de recherche et de participer à la rédaction d'un article scientifique ». À la fin de la phase 3 dite de consolidation, dont la validation sanctionne la certification professionnelle, il doit être en mesure de «s'implique[r] dans les activités académiques et [être] capable de formuler la question d'un sujet de recherche, identifier les objectifs primaires et secondaires et la méthodologie pour y répondre, analyser les résultats, élaborer et en proposer une interprétation ».

\section{Un ensemble de dispositifs hétérogènes}

En dépit d'une telle unanimité des intentions et des recommandations prescriptives, la réalité des initiatives effectivement opérationnelles en termes de programmes dédiés à la formation à la recherche est sans doute très hétérogène, notamment au niveau du cursus prégradué des études. Un guide de l'Association for Medical Education in Europe (AMEE), publié en 2012 [12], identifiait plusieurs obstacles au développement des habiletés et des dispositions pour la recherche. Nous en commentons quelquesuns, de façon non exhaustive.

Certains d'entre eux sont liés à la difficulté d'insérer un tel programme dans des curriculums souvent déjà fort surchargés et, corollairement, aux problèmes liés aux coûts induits et à la disponibilité des ressources nécessaires, tant humaines que matérielles. De ce fait, dans beaucoup de facultés, les activités concernées ne sont proposées que dans le cadre d'enseignements optionnels ou d'activités extra-curriculaires (sous forme de stages d'été, par exemple), qui ne concernent pas, dès lors, la totalité des étudiants d'une promotion. En 2009, le GMC a même dû consentir à réduire de 30 à $10 \%$ la part du curriculum qui devait être dévolue aux enseignements optionnels, limitant sans doute encore de ce fait les possibilités d'accès des étudiants à une formation à la recherche.

Des positions plus radicales sont cependant défendues par certaines institutions. Ainsi, la faculté de médecine de l'Université Duke, aux États-Unis, consacre-t-elle la totalité de la troisième année de son programme d'études à des activités académiques visant à diplômer des cliniciens-chercheurs ou des cliniciens-enseignants. De telles initiatives sont en réalité plus proches des réflexions et des initiatives en cours concernant l'opportunité de mettre en place des parcours mixtes, médicaux et scientifiques, selon le modèle des $\ll M D-P h D$ (Medicince Doctor-Philosophice Doctor) programs» nord-américains. Dans ces mêmes colonnes, une contribution avait rendu compte d'expériences pilotes développées dans ce domaine en France [13]. Des constats en demi-teinte, questionnant par exemple le retour sur investissement des parcours MD$\mathrm{PhD}$ sont aujourd'hui établis [14], illustrant certains paradoxes ou certaines ambiguïtés liées au positionnement professionnel et institutionnel des cliniciens-chercheurs (physician-scientists) [15].

C'est souligner la nécessité que les finalités des programmes de formation à la recherche au cours des cursus prégradués et même post-gradués soient clairement formulées et délimitées en termes d'objectifs poursuivis et de compétences visées. Ainsi, si l'on peut admettre, comme le font la plupart des recommandations rappelées précédemment, émanant des organismes prescripteurs, qu'il existe un continuum entre d'une part, la nécessaire formation à la dimension scientifique de l'exercice de la médecine, en lien avec le courant de la médecine fondées sur les faits probants (evidence-based medicine) et, d'autre part, la formation à la pratique de la recherche en tant que telle, il faut reconnaître en même temps que les capacités et ressources nécessaires sont respectivement différentes, tant en nature qu'en degré de maîtrise. Il convient donc de distinguer clairement, par exemple, l'intention de préparer les étudiants à une pratique judicieuse et réflexive de la médecine, intégrant pour ce faire une approche critique et éthique des savoirs scientifiques produits par la recherche, celle visant à apporter des solutions aux multiples fossés perçus entre le champ de la recherche et celui de la pratique soignante, et à rendre l'une et l'autre plus pertinentes, ou encore celle visant à préparer au plus tôt certains étudiants 
à embrasser la carrière de chercheur à temps plus ou moins complet. Chacune de ces différentes ambitions, toutes aussi légitimes, nécessite une réflexion didactique et pédagogique propre, sous peine de se voir dissoute dans des objectifs trop généraux et indifférenciés.

D'autres difficultés concernent la nature des dispositifs curriculaires et pédagogiques à mettre en œuvre. À cet égard, les auteurs du guide de l'AMEE [12] suggèrent que des dispositifs curriculaires intégrés et favorisant l'apprentissage auto-régulé, dès lors qu'ils solliciteraient à la fois des processus de pensée critique, de résolution de problèmes et des habiletés de communication ou de travail en équipe, par exemple dans le cadre de formats apparentés à l'apprentissage par problèmes, constitueraient ipso facto des dispositifs adéquats pour que les étudiants développent les habiletés et les dispositions nécessaires à la recherche. On ne peut sans doute pas nier que de tels environnements académiques soient des contextes plus favorables que ceux qui recourent encore majoritairement à des approches transmissives ou qui conservent des cloisonnements disciplinaires. Il faut pourtant dénoncer les illusions que véhiculent les notions de capacités ou de stratégies dites génériques, générales ou encore transversales. De telles conceptions conduisent en effet les auteurs à considérer que les capacités de communication - prises en l'occurrence pour exemple- que développe un étudiant en contexte clinique constitueraient, une fois pour toutes, un bagage adéquat pour communiquer en contexte de recherche et qu'il n'y aurait dès lors pas lieu d'y revenir spécifiquement, arguant du même coup que la nécessité de former à la recherche ne justifie pas obligatoirement l'addition de nouveaux dispositifs pédagogiques. Monteiro et al. [16] rappellent toutefois à cet égard judicieusement, et une fois de plus, qu'une accumulation de résultats de recherche en sciences cognitives ont montré la faible puissance des stratégies générales et documenté, à l'inverse, la primauté et le caractère crucial d'un bagage de connaissances et d'autres ressources éminemment spécifiques, mobilisables en contexte. Il faut ainsi se garder de l'idée que des capacités transversales pourraient se construire indépendamment de contextes et de contenus spécifiques.

\section{Quelques principes directeurs}

Pour le dire autrement et plus simplement, si l'on veut outiller adéquatement les étudiants pour qu'ils soient en mesure de construire et résoudre des problèmes de recherche, il faut les exposer au plus tôt, de façon itérative, dans le cadre de dispositifs contextualisés, à des situations qui seront exploitées pour qu'ils construisent et résolvent des problèmes spécifiques de recherche en s'efforçant de réunir les conditions qui favorisent le transfert des apprentissages.

Une telle exigence peut être satisfaite de multiples manières. D'un point de vue pragmatique, un guide formule par exemple douze conseils visant à aider les étudiants à s'insérer dans des environnements de recherche pertinents [17]. De façon moins concrète mais plus structurante, Healey et Jenkins [18] proposent d'articuler la réflexion autour de deux axes permettant de décrire l'intégration de la recherche dans les cursus prégradués. Le premier invite à situer de quelle manière la recherche est présentée dans le curriculum, respectivement plutôt en tant que corpus de contenus et de savoirs, ou plutôt en tant que problèmes à résoudre et processus à mettre en œuvre. Le second invite à considérer la place effectivement offerte aux étudiants, d'un côté plutôt comme spectateurs de la recherche, de l'autre comme participants actifs à la recherche. On pourrait y ajouter qu'il s'agit aussi d'examiner dans le cadre de quelles tensions sont examinées les capacités concernées, en lien non seulement avec le champ de la méthodologie mais, d'abord, avec celui de l'épistémologie qui a trait aux conditions de construction des connaissances au regard de leur statut présumé, leur genèse et leur forme, d'où découle leur valeur [19].

$\mathrm{Au}$ total, la problématique de la formation à la recherche au cours de la formation initiale, et tout particulièrement $\mathrm{du}$ cursus prégradué, ne peut pas se satisfaire, pour solde de tout compte, de pétitions générales de principe, arguant de l'importance de la formation scientifique pour une pratique raisonnée de la médecine. Elle nécessite que les enjeux soient clairement identifiés, que les compétences visées soient explicitement délimitées, que les situations professionnelles concernées soient précisément reconnues, et que des dispositifs curriculaires et pédagogiques appropriés soient planifiés. Ce ne sont pas là de minces défis mais l'article de Zhang et al. [1] illustre opportunément que la mise en œuvre de dispositifs pertinents ne mobilise pas forcément des ressources académiques disproportionnées, dès lors que les finalités en ont été bien définies.

\section{Jean JOUQUAN ${ }^{*}$}

Rédacteur en chef

*Mailto : jean.jouquan@univ-brest.fr

\section{Références}

1. Zhang W, Cambier C, Zhang Y, Detroz P, Farnir F, Gustin $\mathrm{C}$ et al. Développer les compétences en recherche chez les étudiants en médecine: une intervention éducative d'apprentissage en équipe. Pédagogie Médicale 2019. DOI: $10.1051 /$ pmed $/ 2019021$.

2. European Higher Education Area and Bologna Process (EHEA). The framework of qualifications for the European higher education area. 2018. Disponible sur http://www. ehea.info/media.ehea.info/file/2018 Paris/77/8/EHEA Paris2018_Communique_AppendixIII_952778.pdf.

3. World Federation for Medical Education (WFME). Global standards for quality improvement in medical education. Basic medical education WFME global standards for quality improvement. The 2015 Revision. 2015. Disponible sur https://wfme.org/download/wfme-global-standardsfor-quality-improvement-bme/.

4. General Medical Council. Outcomes for graduates (Tomorrow's Doctors). Mapping-2009-to-2017-and-GPC-. London: GMC, 2015. Disponible sur https://www.gmc-uk.org/ education/standards-guidance-and-curricula/standardsand-outcomes/outcomes-for-graduates. 
5. General Medical Council. Outcomes for graduates (Tomorrow's Doctors). London: GMC, 2015. Disponible sur https://www.gmc-uk.org/-/media/documents/outcomesfor-graduates-jul-15-1216_pdf-61408029.pdf.

6. General Medical Council. Generic professional capabilities framework. Manchester: GMC, 2017. Disponible sur https://www.gmc-uk.org/education/standards-guidanceand-curricula/standards-and-outcomes/generic-professio nal-capabilities-framework.

7. Comité d'agrément des facultés de médecine du Canada (CAFMC). Normes d'agrément des programmes d'éducation médicale en vue de l'obtention d'un diplôme en médecine (MD). 2019. Disponible sur https://cacmscafmc.ca/sites/default/files/documents/Normes_Élé ments_CAFMC_AU 2020-2021.pdf.

8. Frank JR, Snell L, Sherbino J, Boucher A (Rédacteurs). Référentiel de compétences CanMEDS 2015 pour les médecins. Ottawa: Collège royal des médecins et chirurgiens du Canada, 2015. Disponible sur http://canmeds. royalcollege.ca $/ \mathrm{fr} /$ referentiel.

9. Michaud PA, Jucker-Kupper P, Members of the Profiles working group. PROFILES/Principal Objectives and Framework for Integrated Learning and Education in Switzerland. Bern: Joint Commission of the Swiss Medical Schools, 2017. Disponible sur http://www.profilesmed.ch/ doc/Profiles 2017.pdf.

10. Ministère de l'enseignement supérieur et de la recherche. Études médicales Régime des études en vue du premier et du deuxième cycle. Bulletin officiel 2013;20. Disponible sur https://www.enseignementsup-recherche.gouv.fr/pid20536/ bulletin-officiel.html.
11. Ministère de l'éducation nationale, de l'enseignement supérieur et de la recherche. Arrêté du 21 avril 2017 relatif aux connaissances, aux compétences et aux maquettes de formation des diplômes d'études spécialisées et fixant la liste de ces diplômes et des options et formations spécialisées transversales du troisième cycle des études de médecine. Journal officiel de la République française 2017. Disponible sur https://www.legifrance. gouv.fr/.

12. Laidlaw A, Aiton J, Struthers J, Guild S. Developing research skills in medical students: AMEE Guide No. 69. Med Teach 2012;34:754-71.

13. Blaise BJ, Claris O, Emsley L, Étienne J, Gilly F-N, Samarut J et al. Un «MD-PhD program» au sein de l'Université de Lyon. Pédagogie Médicale 2012;13: 273-83.

14. Wolfson RK, Arora VM. The physician-scientist pipeline: Return on investment. Med Educ 2018;52:462-8.

15. Rowland P, Ng S. Multiple boundaries: Professional and institutional identities of clinician-scientists. Med Educ 2017;51:566-72.

16. Monteiro S, Sherbino J, Sibbald M, Norman G. Critical thinking, biases and dual processing: The enduring myth of generalisable skills. Med Educ 2019. DOI: 10.1111/medu.13872.

17. Mabvuure NT. Twelve tips for introducing students to research and publishing: A medical student's perspective. Med Teach 2012;34:705-9.

18. Healey M, Jenkins A. Developing undergraduate research. York: The Higher Education Academy, 2009.

19. Piaget J (Dir.). Logique et connaissance scientifique. Paris : Gallimard (Encyclopédie de la Pléiade), 1967. 